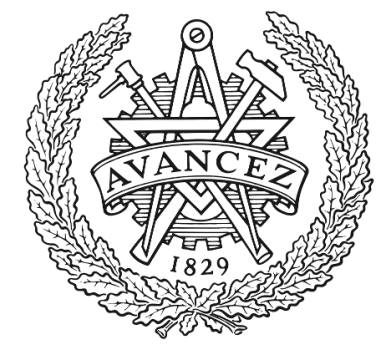

CHALMERS

UNIVERSITY OF TECHNOLOGY

\title{
Nanoplasmonic-nanofluidic single-molecule biosensors for ultrasmall sample volumes
}

Downloaded from: https://research.chalmers.se, 2023-04-26 15:31 UTC

Citation for the original published paper (version of record):

Spodnivakova, B., Jungová, H., Käll, M. et al (2021). Nanoplasmonic-nanofluidic single-molecule biosensors for ultrasmall sample volumes. ACS Sensors, 6(1): 73-82.

http://dx.doi.org/10.1021/acssensors.0c01774

N.B. When citing this work, cite the original published paper. 


\title{
Nanoplasmonic-Nanofluidic Single-Molecule Biosensors for Ultrasmall Sample Volumes
}

\author{
Barbora Špačková,* Hana Šípová-Jungová, Mikael Käll, Joachim Fritzsche, and Christoph Langhammer*
}

Cite This: ACS Sens. 2021, 6, 73-82

Read Online

ABSTRACT: Detection of small amounts of biological compounds is of ever-increasing importance but also remains an experimental challenge. In this context, plasmonic nanoparticles have emerged as strong contenders enabling label-free optical sensing with single-molecule resolution. However, the performance of a plasmonic single-molecule biosensor is not only dependent on its ability to detect a molecule but equally importantly on its efficiency to transport it to the binding site. Here, we present a theoretical study of the impact of downscaling fluidic structures decorated with plasmonic nanoparticles from conventional microfluidics to nanofluidics. We find that for ultrasmall picolitre sample volumes, nanofluidics enables unprecedented binding characteristics inaccessible with conventional microfluidic devices, and that both detection times and number of detected binding events can be improved by several orders of magnitude. Therefore, we propose nanoplasmonic-nanofluidic biosensing platforms as an efficient tool that paves the way for label-free single-molecule detection from ultrasmall volumes, such as single cells.

KEYWORDS: biodetection, nanofluidics, single-molecule detection, mass-transport, single-particle plasmonic sensing, ultrasmall volume

$\mathrm{D}$ etection of tiny amounts of chemical and biological entities is one of the most important tasks in chemistry, biology, medicine, environmental monitoring, and homeland security. Optical sensors based on plasmonic nanoantennas supporting localized surface plasmon resonance (LSPR) are promising to provide a solution for this challenge since they combine high sensitivity with label-free detection and vast miniaturization potential. ${ }^{1}$ Furthermore, by utilizing individual plasmonic nanoparticles (NPs) as highly sensitive probes, the detection of single biomolecule binding events has been demonstrated in multiple studies, ${ }^{2}$ as well as in a highly multiplexed fashion. ${ }^{3}$ From an application perspective, singlemolecule detection is particularly important when only a limited sample volume is available and/or when only a very small number of biomolecules are present in the sample. ${ }^{4}$ This is, for instance, relevant for the study of single cells that have a volume of a few picolitres only. ${ }^{5}$ Such single-cell analysis enables studies of intracellular variability in many biological processes and it has been hallmarked as a necessity for progress in cell biology and tissue engineering. ${ }^{6}$ However, studies at the single-cell level require the capacity to manipulate small sample volumes down to a picolitre and the capacity to detect extremely low concentrations of target molecules. ${ }^{7,8}$ To this end, plasmonic biosensors combined with a microfluidic device have recently been used for the detection of single-cell cytokine expression. ${ }^{9}$

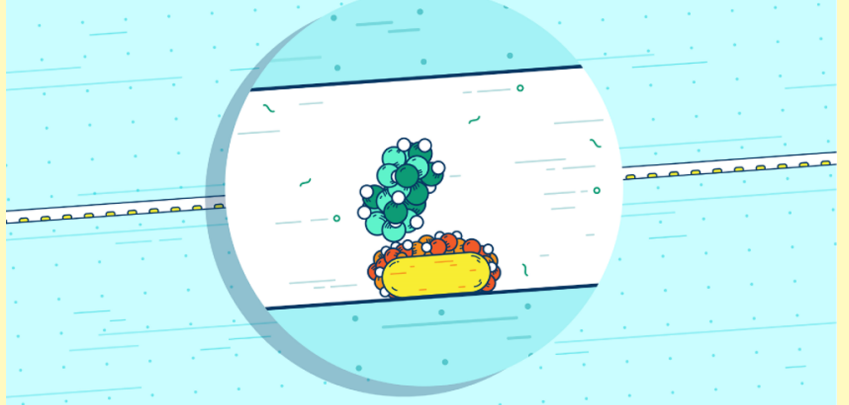




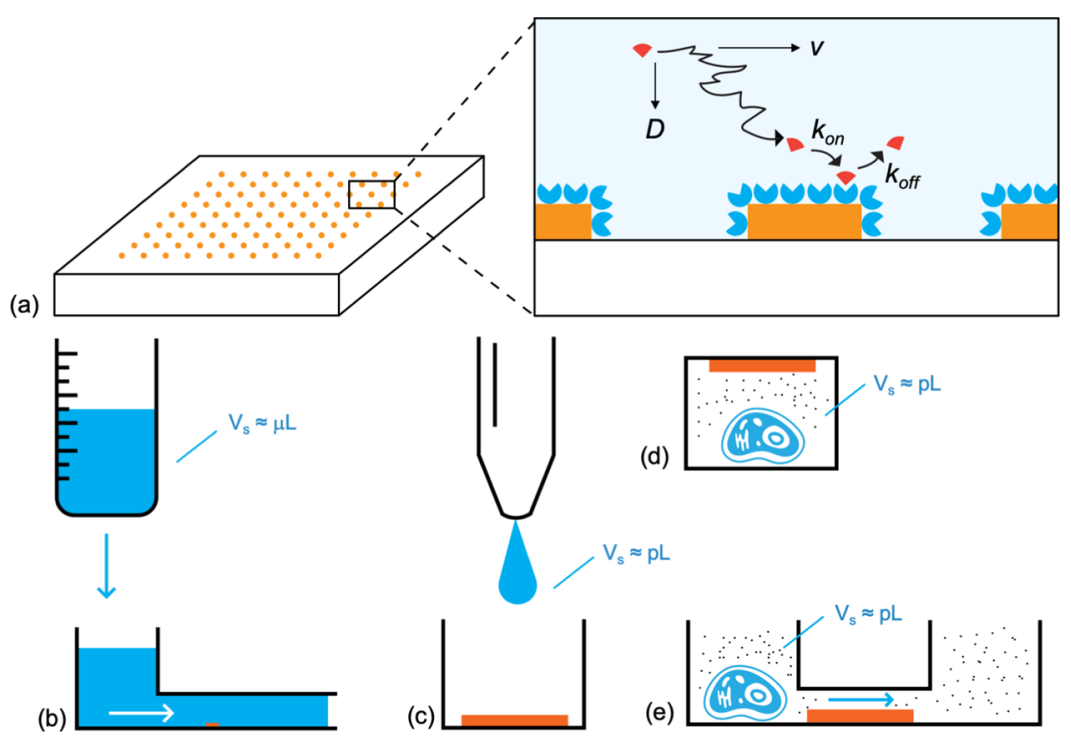

Figure 1. (a) Schematic depiction of a nanoplasmonic biosensor comprising an array of plasmonic NPs functionalized with analyte-specific receptors. The transport of the analyte molecules present in the sample volume to the surface of the particle is defined by the flow velocity, $\mathbf{v}$, and the diffusivity, $D$. The binding and unbinding of the analyte molecules are defined by the kinetic rate constants $k_{\text {on }}$ and $k_{\text {off }}$, respectively. (b-e) Typical scenarios related to single-molecule detection: (b) a large sample volume is flown through a fluidic channel, (c) an ultrasmall sample volume is pipetted into a fluidic well, and (d) a single cell is inserted into a fluidic well. The cell can then either be lysed to enable subsequent analysis of the intracellular content or secrete metabolites that are analyzed and (e) a single cell is immobilized inside a fluidic structure adjacent to a nanofluidic channel and the intracellular content or its metabolites are transported into the sensing region, where they are detected.

on binding kinetics. In particular, using both an analytical approach and stochastic simulations of molecular mass transport, we show that for applications where ultrasmall sample volumes are critical, such as in single-cell analysis, efficient mass transport to the plasmonic sensor still remains a considerable challenge. Moreover, we propose a solution to this problem by combining nanoplasmonic sensing with nanofluidics into a single device. Nanofluidics provides an efficient tool for the handling of fluids in the subpicoliter range by downsizing the fluid control to the nanoscale. ${ }^{14}$ However, apart from proof-of-principle studies demonstrating the feasibility of nanoplasmonic-nanofluidic biosensing, ${ }^{15,16}$ it has so far mostly been used in the context of fluorescence microscopy. ${ }^{17,18}$ As the key result of our study, we identify a regime where the downscaling of the fluidic system from widely used conventional microfluidics to the dimensions characteristic of nanofluidic structures enables the reliable plasmonic label-free detection of single molecules within a realistic detection time range that has not been accessible previously.

\section{MASS TRANSPORT IN NANOPLASMONIC BIOSENSORS}

In this study, we assume a nanoplasmonic biosensor comprising a fluidic structure containing a solution of analyte molecules that is transported via diffusion and convection when pressure-driven flow is induced through the structure. One of the walls of the fluidic structure is decorated with an array of plasmonic NPs functionalized with immobilized receptors with a surface density, $\Gamma_{0}$ (Figure 1$)$. We assume that the analyte molecules exclusively bind to the receptors (i.e., there is no unspecific binding) in a 1:1 ratio and the interaction is characterized by the association and dissociation constants, $k_{\text {on }}$ and $k_{\text {off }}$. It has to be noted, that this represents an idealized situation, where unspecific binding is not taken into account. In practice, suppression of unspecific binding represents a considerable challenge, especially for biosensing in complex media. However, we propose that it can be efficiently mitigated using established biochemical surface functionalization methods. ${ }^{19}$ Moreover, we assume that the array of plasmonic NPs, as well as the optical readout, is designed in a way that single-binding and unbinding events can be resolved. In this setting, the binding and unbinding of analyte molecules is controlled by the complex interplay between convection, diffusion, and reaction of the analyte with the immobilized receptors and can be described by the convection-diffusion equation coupled with the first-order Langmuir kinetic equation

$$
\begin{aligned}
& \frac{\partial c}{\partial t}=D \nabla^{2} c-\mathbf{v} \cdot \nabla c \\
& \frac{\mathrm{d} \Gamma}{\mathrm{dt}}=k_{\mathrm{on}} c_{S}\left(\Gamma_{0}-\Gamma\right)-k_{\mathrm{off}} \Gamma
\end{aligned}
$$

where $D$ is the diffusivity of the analyte molecule, $\mathbf{v}$ is the flow velocity field defined by the architecture of the fluidic structure, $c$ is the (bulk) concentration of the analyte molecules inside the fluidic structure, $c_{\mathrm{S}}$ is the (bulk) concentration of unbound analyte molecules at the NP surface, and $\Gamma$ is the surface concentration of the bound analyte molecules.

In the following sections, we have explored the binding characteristics for a series of typical scenarios related to singlemolecule detection by focusing on the impact of characteristic dimensions of the used fluidic structures to enable the direct comparison of micro- and nanofluidics in this respect. As we will demonstrate further, the key characteristic that leads to qualitatively different conclusions is the sample volume (volume of the analyte solution, $V_{\mathrm{s}}$ ) relative to the volume of the fluidic structure. Therefore, each section of our work reported below describes a different scenario in this respect. Section 2 analyzes the situation that considers an infinite sample volume that flows through a fluidic channel. In practice, 


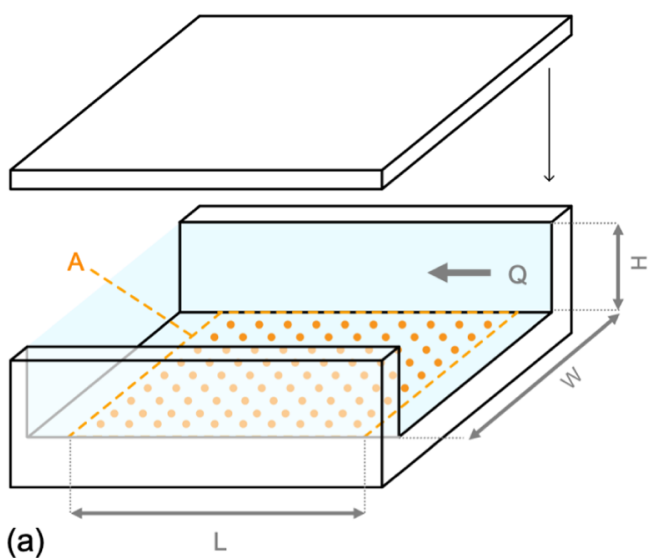

(b) $\begin{array}{ll}\Gamma_{\mathrm{p}}=0.04 \mathrm{NPs} \cdot \mu \mathrm{m}^{-2} & \mathrm{NP} \text { size }=30 \times 30 \times 80 \mathrm{~nm} \\ D=10 \mu \mathrm{m}^{2} \cdot \mathrm{s}^{-1} & \Gamma_{0}=10^{-13} \mathrm{~mol} \cdot \mathrm{mm}^{-2}\end{array}$

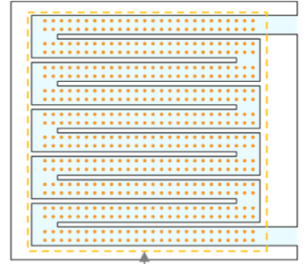

Field of view:

(c) $100 \mu \mathrm{m} \times 100 \mu \mathrm{m}$

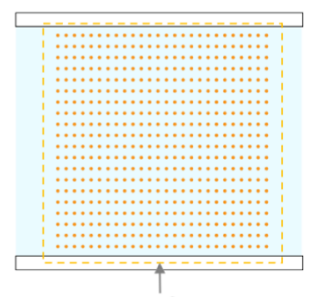

Field of view:

(d) $100 \mu \mathrm{m} \times 100 \mu \mathrm{m}$

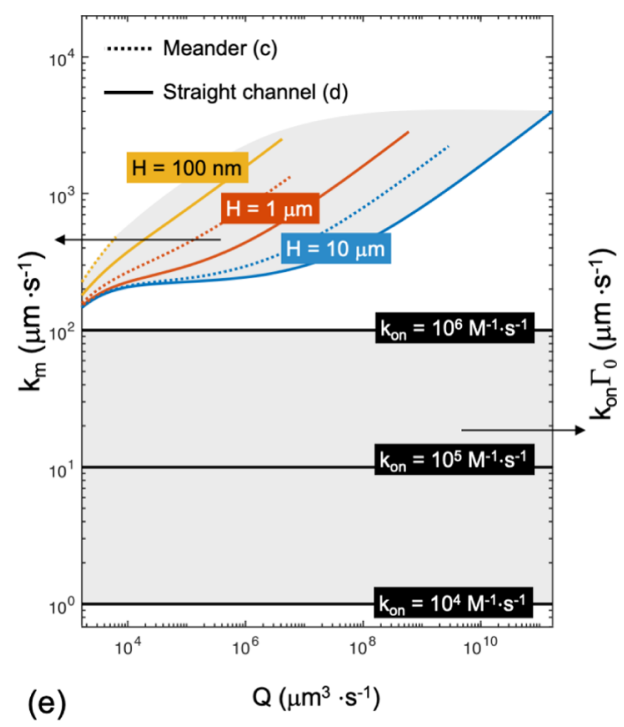

(e)

Figure 2. (a) Schematic depiction of the simulated system consisting of an infinite sample volume, which flows at a volumetric flow rate $Q$ through a sensing region of a fluidic channel of height $H$, width $W$, and length $L$, comprising a functionalized plasmonic NP array of area $A$. (b) Summary of the parameters used in the calculations. $(c, d)$ Top view of the two investigated configurations of the fluidic channel: (c) meandering channel with $L=1000 \mu \mathrm{m}$ and $W=10 \mu \mathrm{m}$ and (d) straight channel with $L=W=100 \mu \mathrm{m}$. Both types of fluidic systems contain the same NP array defined by the area $A=100 \times 100 \mu \mathrm{m}^{2}$, i.e., the field of the view of a $100 \times$ microscope objective. (e) Calculated comparison of $k_{\mathrm{m}}$ and $k_{\mathrm{on}} \Gamma_{0}$ describing the relative rate of reactive capture with respect to the rate of analyte transport for both the meandering channel (dashed lines) and the straight channel (solid lines). The results shown for $k_{\mathrm{m}}$ correspond to a range of technically relevant flow rates $Q$ a specific size of analyte molecules defined by $D$, and they were calculated for the two channel types assuming the channel height $H$ ranging from $10 \mu \mathrm{m}$ characteristic of microfluidics down to 100 $\mathrm{nm}$ characteristic of nanofluidics. The results shown for $k_{\mathrm{on}} \Gamma_{0}$ correspond to the values characteristic for protein interactions, that is, $k_{\mathrm{on}}=10^{4}-10^{6}$ $\mathrm{M}^{-1} \cdot \mathrm{s}^{-1}{ }^{13}$ Note that for the entire range of assumed parameters $k_{\mathrm{m}}>k_{\mathrm{on}} \Gamma_{0}$, which means that the system is predominantly reaction-limited.

this situation can be encountered when a sample is taken from a patient and flushed through the fluidic structure (Figure $1 \mathrm{~b}$ ). The volume is large enough (i.e., $\mu \mathrm{L}$ or $\mathrm{mL}$ ) that the detection time is not limited by the time needed to flow the whole volume through the fluidic system. ${ }^{20}$ Sections 3 and 4 analyze a different scenario that considers a finite sample volume, containing only a limited number of molecules, where the means of loading the sample into the device is critical. In practice, this situation corresponds to applications dealing with ultrasmall sample volumes ( $\mathrm{pL}$ or $\mathrm{nL}$ ). Specifically, Section 3 considers a confined finite sample volume in an enclosed fluidic well without flow. This situation is encountered when a tiny amount of a sample is pipetted inside a well, followed by enclosing the well (Figure 1c); alternatively, a single cell is inserted into the fluidic well, and then the cell can be either lysed and intracellular content is subsequently analyzed ${ }^{9}$ or its metabolite secretion is studied ${ }^{21,22}$ (Figure 1d). Section 4 considers a finite sample volume that flows through a fluidic channel. This scenario corresponds to a situation where, for e.g., a single cell is immobilized close to a nanofluidic channel and the intracellular content or its metabolites are transported into the nanofluidic sensing region, where they are detected ${ }^{7}$ (Figure 1e).

\section{INFINITE SAMPLE VOLUME}

In this section, we consider the first case of an infinite sample volume that flows through a fluidic channel with a volumetric flow rate, $Q$ (Figure 2a). For this scenario, the problem of mass transport described by eqs 1 and 2 can be treated analytically in terms of the two compartment model, ${ }^{23,24}$ which states that when binding occurs quasi-steadily, the analyte concentration at the surface can be described as

$$
\frac{c_{\mathrm{s}}}{c_{0}}=\frac{1}{1+k_{\mathrm{on}} \Gamma_{\mathrm{f}} / k_{\mathrm{m}}}
$$

where $\Gamma_{\mathrm{f}}=\Gamma_{0}-\Gamma$ is the surface concentration of the free receptors, $c_{0}$ is the initial (bulk) concentration of the analyte molecules, and $k_{\mathrm{m}}$ is the mass transfer coefficient, which can be calculated analytically (see SI Section 1) via parameters related to both the fluidic conditions and the architecture of the NP array. ${ }^{25}$ The term in the denominator, the Damköhler number $k_{\mathrm{on}} \Gamma_{\mathrm{f}} / k_{\mathrm{m}}$, describes the relative rate of analyte reactive capture with respect to the rate of analyte transport to the sensor surface. In a regime when $k_{\mathrm{m}} \ll k_{\mathrm{on}} \Gamma_{\mathrm{f}}$, the collection rate is dictated mainly by the transport of the analyte molecules to the binding sites, i.e., the system is diffusion-limited. In the opposite regime, when $k_{\mathrm{m}} \gg k_{\mathrm{on}} \Gamma_{\mathrm{f}}$, mass transport supplies analyte molecules more quickly than the receptors can bind them, the system is therefore reaction-limited. In essence and at the general level, this means that for each specific affinity format (defined by $k_{\text {on }}, k_{\text {off }}, \Gamma_{0}$ of the used molecules) the maximum collection rate is achieved when the system is reaction-limited.

For our analysis, we define our system such that the dimensions of the sensing region of the fluidic channel are given by its height $H$, width $W$, and length $L$. The sensing region comprises the functionalized NPs that are defined by their surface area $A_{\mathrm{p}}$ and their shape. The NPs are arranged into an array with a surface density $\Gamma_{\mathrm{p}}$, covering the area $A=$ $100 \times 100 \mu \mathrm{m}^{2}$ that fits the field of view of a $100 \times$ microscope objective. To implement this scenario, we consider two different arrangements of the fluidic channel: (i) a meandering channel with $W=10 \mu \mathrm{m}$ and $L=1000 \mu \mathrm{m}$ (Figure 2c) and (ii) a straight channel with $W=L=100 \mu \mathrm{m}$ (Figure 2d). We note that the thickness of the walls between the meandering 


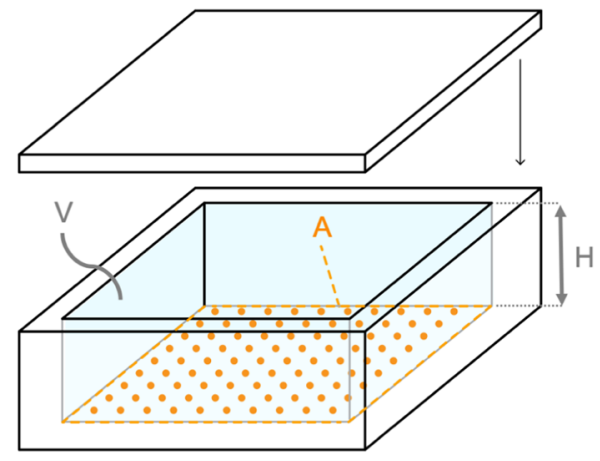

(a)

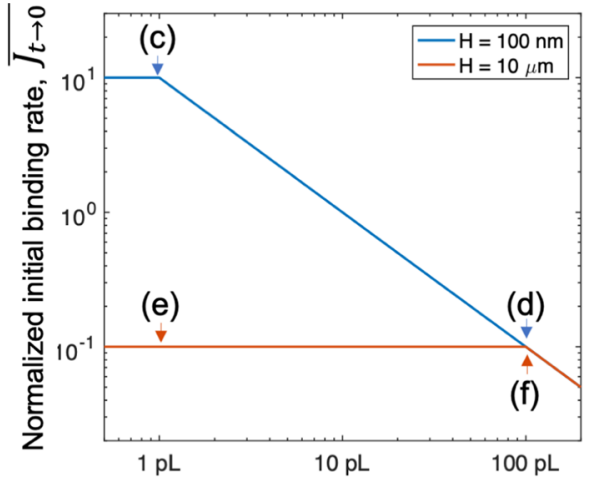

(b)

Volume of the well, $\mathrm{V}$

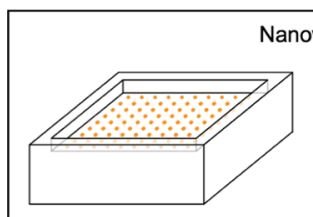

(c) $A=100 \times 100 \mu$

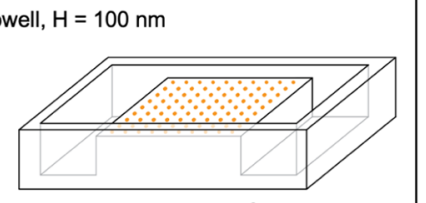

(d) $\mathrm{A}=100 \times 100 \mu \mathrm{m}^{2}$

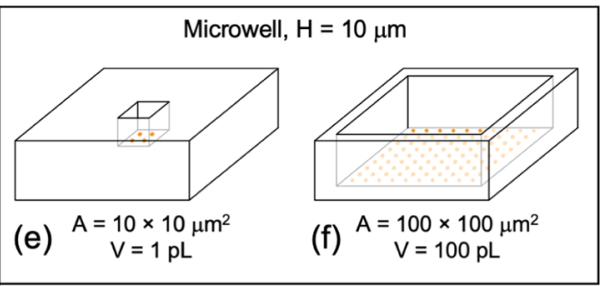

Figure 3. (a) Schematic depiction of the simulated system considering a finite sample volume, $V_{\text {s }}$, placed inside a fluidic well with a volume $V$ and height $H$, decorated with an array of functionalized plasmonic NPs covering an area $A$. (b) Dependency of the normalized initial collection rate (eq 6) on the height and volume of the fluidic well in the reaction-limited regime. The maximal $A$ is limited by $A_{\mathrm{fw}}=100 \times 100 \mu \mathrm{m}^{2}$, i.e., the field of view of a $100 \times$ microscope objective. ( $\mathrm{c}-\mathrm{f})$ Schematic depictions of the investigated fluidic wells defined by their heights $(\mathrm{c}, \mathrm{d}) \mathrm{H}=100 \mathrm{~nm}$ (nanowell) and (e, f) $H=10 \mu \mathrm{m}$ (microwell) and by their volumes (c, e) $V=1 \mathrm{pL}$ and (d, f) $V=100 \mathrm{pL}$.

channels is omitted in the theoretical analysis. In practice, using state-of-the-art nanofabrication, wall thicknesses down to $300 \mathrm{~nm}$ are easily feasible due to well-established vertical etch processes, ${ }^{26}$ thereby not significantly impacting the conclusions from our calculations. To investigate the analyte transport to the binding sites, we have calculated the mass transfer coefficient, $k_{\mathrm{m}}$, and compared it to values of $k_{\mathrm{on}} \Gamma_{0}$ typical for protein interactions (Figure 2e). ${ }^{13}$ Specifically, we carried out calculations for a NP array with parameters previously efficiently used for single-molecule biosensing ${ }^{3}$ (Figure 2b) and for the two types of fluidic systems depicted in Figure 2c,d, by systematically varying the channel height $H$ from $10 \mu \mathrm{m}$, as characteristic of microfluidics, down to $100 \mathrm{~nm}$, as characteristic of nanofluidics, and by screening a range of technically relevant flow rates $Q .{ }^{27}$ Details of the calculations, as well as the results of the extended analysis for a range of scenarios characteristic for a broad set of parameters pertaining to the fluidic structure $(H, W, L, Q), \mathrm{NP}$ array, $\left(A, A_{\mathrm{p}}, \Gamma_{\mathrm{p}}\right)$, and analytes $\left(D, k_{\text {on }}\right)$, can be found in SI Section 1 and Figures $S-1$, $S-2$. We note that for all of the assumed parameters that are relevant for current state-of-the-art single-molecule nanoplasmonic biosensors, $k_{\mathrm{m}} \gg k_{\mathrm{on}} \Gamma_{0}$ can be achieved for high enough, but still technically relevant, $Q$. This also means that the collection rate can reach its maximum, i.e., the reactionlimited regime, irrespective of the fluidic structure dimensions. Thus, $c_{\mathrm{s}} \approx c_{0}$ (eq 3 ), and the collection rate (number of bound analyte molecules per unit time) can be written as

$$
J=\frac{\mathrm{d} N}{\mathrm{~d} t} \approx k_{\mathrm{on}} c_{0}\left(\Gamma_{0} A_{\mathrm{s}}-\mathrm{N}\right)-k_{\mathrm{off}} N
$$

by using eq 2 where $A_{\mathrm{s}}=\Gamma_{\mathrm{p}} A_{\mathrm{p}} A$ represents the active sensing area, that is, the area covered by receptors. From eq 4 it can be seen that in the reaction-limited regime, for any specific affinity format (defined by $k_{\mathrm{on}}, k_{\mathrm{off}}$, and $\Gamma_{0}$ ) and any specific analyte concentration, the collection rate can only be improved by enlarging the active sensing area. However, the size of the active sensing area is limited by a series of constraints related to the optical readout for single-molecule detection: (i) The maximal surface area of the NPs in the array $\left(A_{\mathrm{p}}\right)$ is limited by the condition of single-molecule resolution since the optical response to a single-molecule binding event worsens with increasing dimensions of the plasmonic NP. ${ }^{28}$ (ii) The maximal area of the NP array $(A)$ is limited by the field of view of the used microscope. (iii) The maximal density of the NP array $\left(\Gamma_{\mathrm{p}}\right)$ is limited by the optical resolution of the microscope, i.e., the response from each individual NP has to be resolved separately, avoiding incidental cross-talk between neighboring NPs.

In other words, and as the main conclusion for a fluidic channel connected to an infinite reservoir of the analyte, changing the channel dimension from the micro- to the nanofluidic regime has little to no impact on the collection rate under predominantly reaction-limited conditions and, therefore, neither offers a handle to significantly improve the overall binding characteristics nor the sensor response time.

\section{FINITE SAMPLE VOLUME INSIDE A FLUIDIC WELL}

The situation described above becomes very different when we consider a scenario where the sample volume, $V_{s}$, is very small, that is, in the $\mathrm{pL}$ range and thus below the volumes that effectively can be handled by traditional microfluidics ( $\mu \mathrm{L}$ or $\mathrm{mL}$ ). In this regime, as we will illustrate in detail, the characteristic dimensions of the fluidic system have a significant impact on the binding characteristics and thus on the critical timescales for single-molecule detection. In the following example, we assume the finite sample volume $\left(V_{s}\right)$ containing a finite number of molecules, $M_{0}=c_{0} V_{s}$, that is placed in an enclosed fluidic well under stagnant conditions 


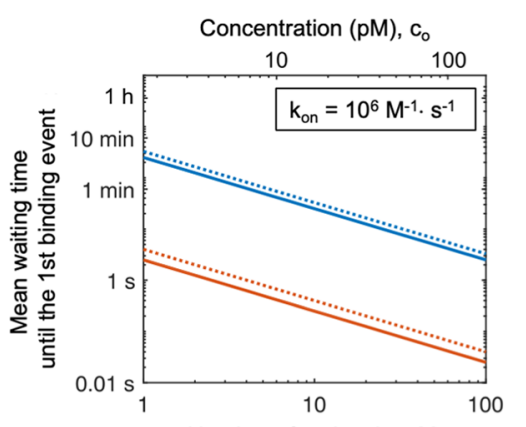

(a)

Number of molecules, $M_{0}$

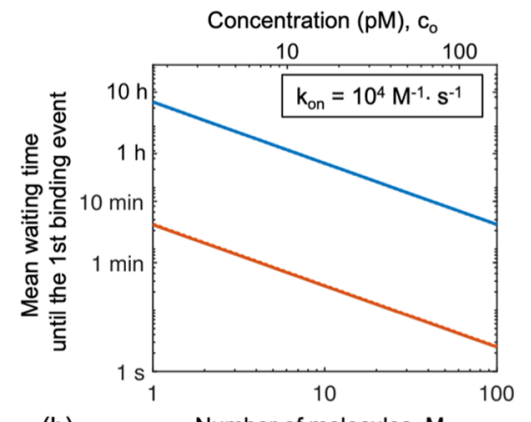

(b)

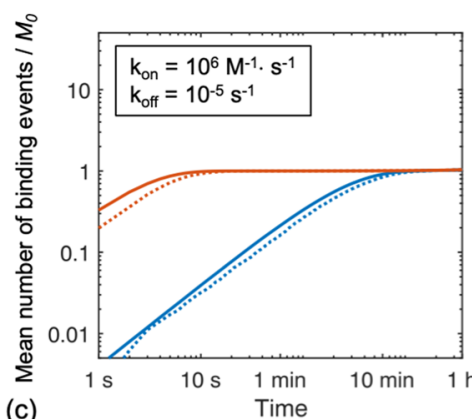

(c)

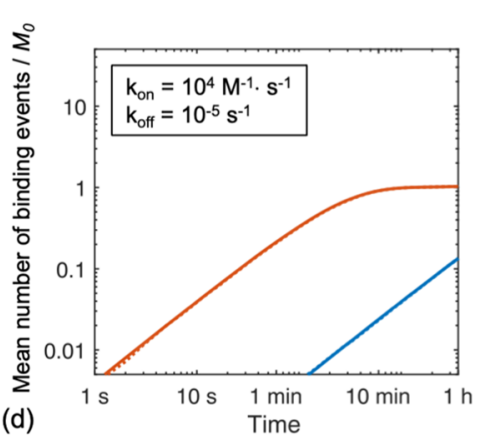

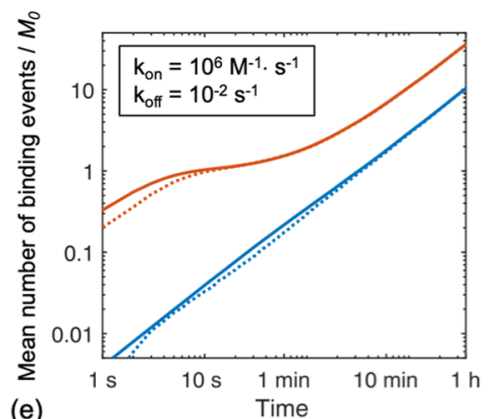

(e)

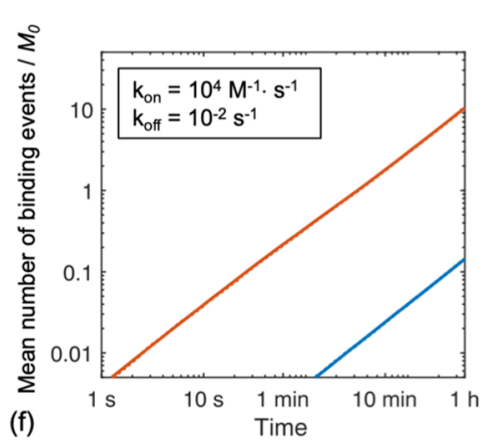

- Microwell - analytical model …..... Microwell - stochastic model

........ Nanowell - stochastic mode

- Nanowell - analytical model

Figure 4. Binding characteristics of $1 \mathrm{pL}$ sample volume confined inside a microwell and a nanowell with defined heights, $H=10 \mu \mathrm{m}$ and $H=100$ $\mathrm{nm}$, respectively. The architecture of the wells corresponds to those described in Figure $3 \mathrm{e}$ for the microwell and Figure $3 \mathrm{c}$ for the nanowell. The parameters corresponding to the NP array and molecules are summarized in Figure 2b. (a, b) Dependency of the mean waiting time until the first binding event on the number of the analyte molecules present in a $1 \mathrm{pL}$ sample volume, $M_{0}$, at the concentration $c_{0}$. (c-f) Mean number of binding events for each molecule present in a $1 \mathrm{pL}$ volume, $\mathrm{N}^{+} / M_{0}$. The results are shown for the $k_{\text {on }}-k_{\text {off }}$ space of biomolecular interactions typical of proteins.

without applied flow (Figure 3a). The fluidic well is defined by its volume, $V \geq V_{s}$, and height, $H$, and comprises an array of functionalized plasmonic NPs defined in the same manner as in Section 2. It has to be noted that in the situation when $V_{\mathrm{s}}<$ $V$, the analyte molecules are transported into the entire volume by diffusion and the sample is therefore diluted.

The analytical theory of mass transport ${ }^{25}$ used in Section 2 is essentially not applicable for such an enclosed system, as it is valid only for an open system in a quasi-steady state. Therefore, we have instead utilized a stochastic diffusionreaction model that rigorously describes the behavior of molecules inside fluidic wells with functionalized surfaces (details in Methods ). We have carried out simulations for a broad range of parameters pertaining to the dimensions of a fluidic well $(H, V)$, the architecture of an NP array, $\left(A, A_{\mathrm{p}}, \Gamma_{\mathrm{p}}\right)$, and properties of analytes $\left(D, k_{\mathrm{on}}\right)$. As one important general conclusion, the results show (SI Section 2, Figures S-3, S-4) that for all scenarios relevant for current state-of-the-art singlemolecule nanoplasmonic biosensors, the mass transport is mostly reaction-limited. Moreover, the binding characteristics are independent from the vertical or horizontal position of the molecules inside the well at the beginning of the detection process. In other words, the binding characteristics are independent of the sample placement or, in the case $V_{s}<V$, the concentration profile during sample dilution (diffusivity of analyte molecules). Therefore, the concentration of the unbound analyte molecules at the surface of the NPs corresponds to the mean concentration of the unbound analyte molecules inside the fluidic well, i.e., $c_{s}(t)=M_{0} / V-$ $N(t) / V$. Thus, using eq 2 , the collection rate can be written as

$$
J=\frac{\mathrm{d} N}{\mathrm{~d} t} \approx k_{\mathrm{on}}\left(\frac{M_{0}}{V}-\frac{N}{V}\right)\left(\Gamma_{0} A_{\mathrm{s}}-N\right)-k_{\mathrm{off}} N
$$

Equation 5 suggests that for each specific affinity format (defined by $k_{\text {on }}, k_{\text {off }}, \Gamma_{0}$ ), the sample volume and concentration (defined by $M_{0}$ ), the collection rate can be improved by maximizing the $A_{\mathrm{s}} / V$ ratio. In other words, enlarging of the active sensing area $\left(A_{s}\right)$ is beneficial only if it does not require an increase of the volume of the well $\left(V>V_{s}\right)$, as this would lead to further dilution of the sample. Thus, as the key point, in comparison to the case for the infinite sample volume analyzed first, not only the architecture of the NPs, but also the size of the fluidic structure is critical in the present scenario.

To now showcase this scenario explicitly, we consider different volumes, $V$, of a fluidic well that range from subpicolitre to hundreds of picolitre and two fixed heights, a microwell with $H=10 \mu \mathrm{m}$ and a nanowell with $H=100 \mathrm{~nm}$ (Figure $3 c-f$ ). All assumed fluidic wells comprise plasmonic NP arrays with the same $\Gamma_{\mathrm{p}}$ and $A_{\mathrm{p}}$ and the same number of molecules, $M_{0}$. In both cases, we assume that the area of the plasmonic NP array is either limited by $A_{\mathrm{fw}}=100 \times 100 \mu \mathrm{m}^{2}$, the field of view of a $100 \times$ microscope objective, or by the dimensions of the horizontal wall of the fluidic well. To provide a general comparison of binding characteristics that are valid for any number of molecules present in the sample volume, affinity formats and NP arrays, the initial collection rates, $J_{t \rightarrow 0}($ eq $5, N(t \rightarrow 0)=0)$, we thus show as the normalized values (Figure $3 b$ ) 


$$
\overline{J_{t \rightarrow 0}}=\frac{J_{t \rightarrow 0}}{k_{\mathrm{on}} M_{0} \Gamma_{0} \Gamma_{\mathrm{p}} A_{\mathrm{p}}}=\frac{A}{V}
$$

For both the micro- and the nanowell it applies that for $V>$ $H A_{\mathrm{fw}}$ the area of the NP array is limited by the field of view of the microscope and thus the normalized initial collection rates increase with decreasing volume since the $A / V$ ratio increases (eq 6). For $V \leq H A_{\mathrm{fw}}$, the area of the NP array matches the area of the wells' horizontal wall and thus the normalized initial collection rates are constant since the $A / V$ ratio also is constant. As a consequence, the normalized initial collection rates for volumes $V>100 \mathrm{pL}$ are equal for the nanowell (Figure 3d) and the microwell (Figure 3f), since the areas of the NP array are identical and match the field of view of the microscope. However, for volumes $V<100 \mathrm{pL}$, the initial collection rates are remarkably higher for the nanowell (Figure $3 c)$ than for the microwell (Figure 3e), since the area of the NP array inside the nanowell is larger. Therefore, for ultrasmall sample volumes $\left(V_{s}<100 \mathrm{pL}\right)$, the collection rates can be improved by up to 2 orders of magnitude by decreasing the dimensions of the fluidic system from the microfluidic to the nanofluidic range and by matching the volume of the fluidic well to the sample volume. In this way, analyte dilution is avoided, while at the same time the sensing area is maximized. We also note that, analogously to the case of Section 2, binding characteristics are not dependent on the width of the well, as long as the well is able to host the entire NP array (Figure $2 \mathrm{c}, \mathrm{d})$.

To further investigate the impact of the size of a fluidic well on the binding characteristics of an analyte from a finite volume, we have calculated two important characteristics: (i) the mean waiting time until the first detected binding event by the sensor, $t_{1}$, and (ii) the total number of binding events, $N^{+}$, detected as a function time. Using eq 5 , the mean waiting time until the first binding event can be defined as

$$
t_{1}=J_{t \rightarrow 0}^{-1}=\frac{V}{k_{\mathrm{on}} M_{0} \Gamma_{0} A_{\mathrm{s}}}
$$

Please note that this parameter is sometimes referred to as the accumulation time. ${ }^{11}$ Assuming that the number of analyte molecules is much smaller than the number of receptors $\left(N_{0}=\right.$ $\left.\Gamma_{0} A_{\mathrm{s}}\right), M_{0} \ll N_{0}$, eq 5 can be solved analytically, and the number of binding events can be written as

$$
N^{+}=M_{0} K^{2}\left[1-e^{-t\left(\frac{k_{\mathrm{on}} N_{0}}{V}+k_{\text {off }}\right)}\right]+M_{0} K k_{\text {off }} t
$$

where $K^{-1}=1+k_{\text {off }} V /\left(k_{\text {on }} N_{0}\right)$.

Figure 4 shows relevant examples for $t_{1}$ and $N^{+}$for a sample volume $V_{\mathrm{s}}=1 \mathrm{pL}$ placed inside fluidic wells with different heights - a microwell with $H=10 \mu \mathrm{m}$ (Figure 3e,f) and a nanowell with $H=100 \mathrm{~nm}$ (Figure 3c). The parameters defining the NP array and the binding properties of the molecules are the same as in the previous example ( $c f$. Figure $2 \mathrm{~b})$. Using these boundary conditions, we executed calculations that cover the $k_{\text {on }}-k_{\text {off }}$ space of biomolecular interactions typical of proteins with $k_{\text {on }}$ ranging from $10^{4}$ to $10^{6} \mathrm{M}^{-1} \cdot \mathrm{s}^{-1}$ and $k_{\text {off }}$ from $10^{-5}$ to $10^{-2} \mathrm{~s}^{-1}$. Specifically, we compare the results of the analytical model pertaining to the reaction-limited regime (eqs 7 and 8 , Figure $4 a-f$ solid lines) with the results of the corresponding stochastic simulations (Figure $4 \mathrm{a}-\mathrm{f}$ dashed lines). We note that the results from the analytical model and the simulations generally agree very well, except for fast binding kinetics, where the values of $t_{1}$ are slightly higher (Figure 4a) and the values of $\mathrm{N}^{+}$are slightly lower at the beginning of the binding curve (Figure 4c,e). This suggests a moderate diffusion limitation in this regime, which, however, does not affect the general conclusions discussed below. As the key results, from the data it can be seen that $t_{1}$ decreases linearly with increasing concentration of the analyte molecules (i.e., the number of analyte molecules confined inside the volume) and it is 2 orders of magnitude smaller for the nanowell than for the microwell (Figure 4a,b). Specifically, when only a few molecules are confined inside the microwell, $t_{1}$ ranges from minutes (Figure $4 a$ ) to tens of hours (Figure $4 b$ ), depending on $k_{\text {on }}$. As the key point, for most $k_{\text {on }}$ this is far beyond practical timescales. On the other hand, if the same sample containing only a few molecules is confined inside the nanowell instead, $t_{1}$ is substantially reduced to the practically relevant timescales ranging from seconds (Figure 4a) to minutes, depending on $k_{\text {on }}$ (Figure $4 \mathrm{~b}$ ).

Furthermore, we see that the binding rate (number of binding events per time, $\left.\mathrm{dN}^{+} / \mathrm{d} t\right)$ is substantially increased for the nanowell compared to the microwell for the whole $k_{\text {on }}-k_{\text {off }}$ space (Figure $4 \mathrm{c}-\mathrm{f}$ ). Equilibrium is reached 2 orders of magnitude faster, and also the binding rate at equilibrium is 2 orders of magnitude higher for the nanowell. Specifically, for kinetics with low $k_{\text {on }}$ and high $k_{\text {off }}$ it can be seen in Figure $4 \mathrm{f}$ that, while in the microwell each molecule in the sample volume is detected within $1 \mathrm{~h}$ with a probability of $3.5 \%$ (corresponds to $N^{+} / M_{0}=0.0035$ ), in the nanowell, each molecule is detected about 10 times per hour $\left(N^{+} / M_{0}=10\right)$. In other words, not only enables the nanowell faster detection but it actually enables multiple detection events of a single molecule within reasonably short time. The detection limit of plasmonic biosensors with single-molecule resolution, defined in terms of the minimal amount of analyte molecules detected in a reasonable time, is mainly limited by the binding rate. ${ }^{12,13,29}$ This unprecedented performance of the nanowell implies that it, similar to a photon multiplier, can "multiply" the signal from a very small number of analyte molecules and thus, in principle, improve the detection limit significantly. To put this finding into perspective from an application viewpoint, we note that binding kinetics with low $k_{\text {on }}$ and high $k_{\text {off }}$ are a regime characteristic for many biological processes, such as interactions of cell surface proteins. ${ }^{30}$ At the same time, low $k_{\text {on }}$ and high $k_{\text {off }}$ result in a very low surface concentration of bound molecules in equilibrium, which makes their detection very challenging or even impossible for traditional biodetection techniques, such as biosensors based on surface plasmon resonance (SPR), that rely on measuring the mean surface concentration of the bound molecules. ${ }^{31}$ In this context the predicted multiple detection of the same molecule enabled by the nanowell, as the molecule binds and unbinds, constitutes a significant advantage.

\section{FINITE SAMPLE VOLUME INSIDE A FLOW-THROUGH FLUIDIC CHANNEL}

In Section 3, we have demonstrated the potential of nanofluidics in the field of single-molecule detection from ultrasmall sample volumes. However, despite the recent advances in manipulation of such ultrasmall volumes, ${ }^{32}$ enclosing a sample into a nanowell as described in Section 3 is a considerable challenge to realize in practice. In addition, as the size of a cell typically exceeds the height of a nanowell by more than 2 orders of magnitude, in vivo single-cell analysis would be very difficult. Therefore, in this section, we analyze 

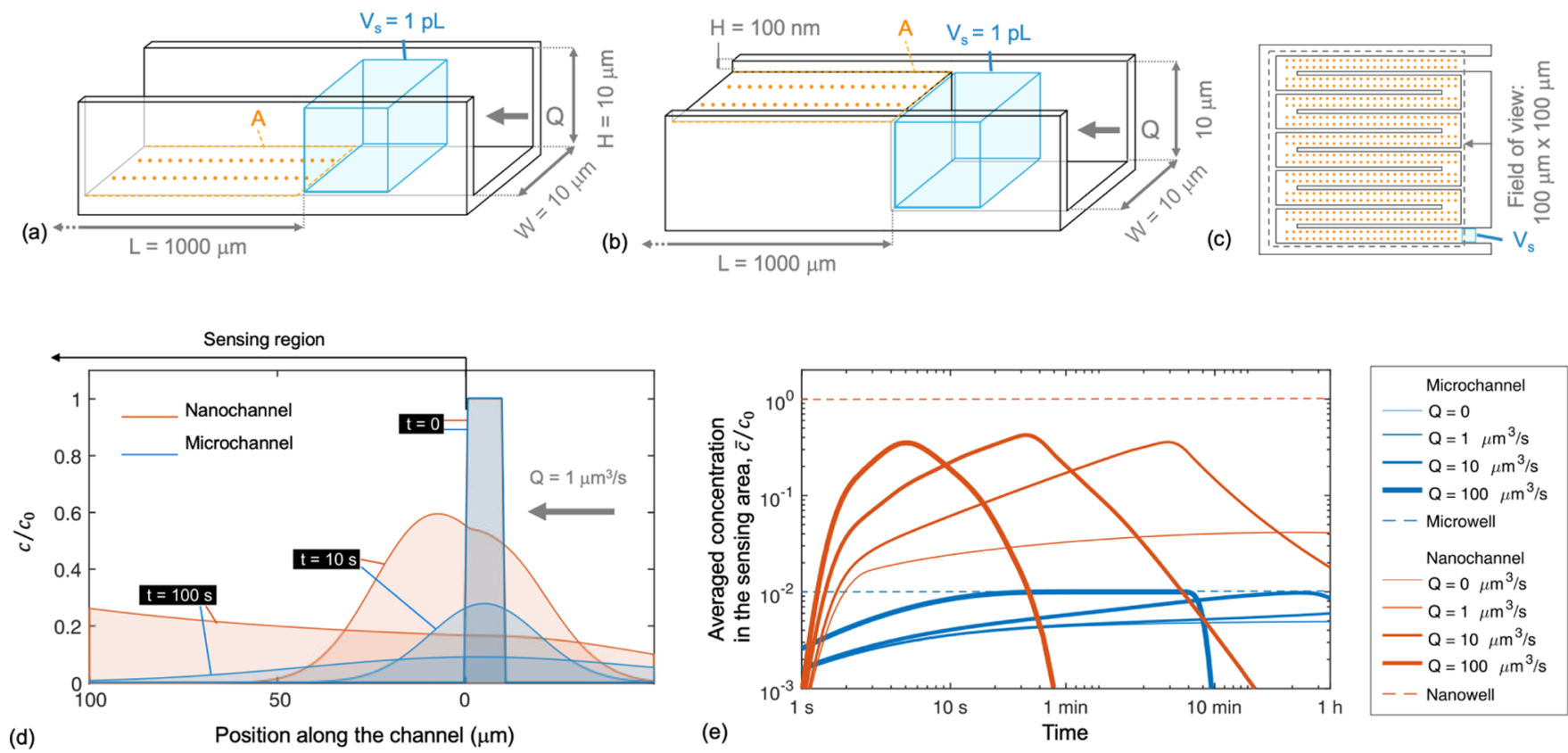

Figure 5. $(\mathrm{a}-\mathrm{c})$ Schematic depiction of the considered system comprising a finite sample volume $V_{\mathrm{s}}=1 \mathrm{pL}$, which flows with a volumetric flow rate $Q$ through (a) a microchannel with $H=10 \mu \mathrm{m}$ or (b) a nanochannel with $H=100 \mathrm{~nm}$. The dimensions of the channels in both cases are $W=10$ $\mu \mathrm{m}$ and $L=1000 \mu \mathrm{m}$ and they are arranged into meanders to fit an array of functionalized plasmonic NPs with an area $A=100 \times 100 \mu \mathrm{m}^{2}$ to match the field of view of a $100 \times$ microscope. At the start of the detection process $(t=0)$, the sample volume is located outside of the sensing region toward which it is transported over time by means of diffusive and convective flow. (c) Top view of the simulated system. (d) Time evolution of the normalized analyte concentration distribution, $c / c_{0}$, for the flow rate $Q=1 \mu \mathrm{m}^{3} \cdot \mathrm{s}^{-1}$ and diffusivity of the analyte $D=10 \mu \mathrm{m}^{2} \cdot \mathrm{s}^{-1}$. (e) Time-dependent normalized averaged concentration over the sensing area, $\bar{c} / c_{0}$. We note that binding and unbinding is not considered here.

the potential benefits of nanofluidics compared to microfluidics in a flow-through configuration. In particular, we assume that the content of an ultrasmall sample volume $V_{s}$ is transported by diffusion and/or convection defined by $D$ and $Q$ respectively, to the sensing region of a micro- or nanofluidic channel, which contains an array of functionalized plasmonic NPs defined in the same manner as in Section 2 (Figure $5 a-c$ ). For this specific configuration, the pertinent equations describing diffusion, convection, and reaction (eqs 1 and 2) cannot be solved analytically. Therefore, we have employed the stochastic diffusion-convection-reaction model, described in Section 6.

To showcase this scenario, we present the results of an analysis of a specific example. However, as discussed below, more general conclusions can be derived based on this example. To relate directly to the results presented in Sections 2 and 3, also here we assume a sample volume $V_{\mathrm{s}}=1 \mathrm{pL}$, which, for example, corresponds to the volume of a $\beta$ cell, ${ }^{33}$ an NP array with $A=100 \times 100 \mu \mathrm{m}^{2}$ with parameters defined in Figure $2 \mathrm{~b}$ and a fluidic channel with the width $W=10 \mu \mathrm{m}$ and length $L=1000 \mu \mathrm{m}$ (Figure 5c). To compare the performance of a nanofluidic with a microfluidic channel, we again compare the two cases of channel heights $H=10 \mu \mathrm{m}$ (microfluidics) and $H=100 \mathrm{~nm}$ (nanofluidics) (Figure 5a,b). Here, we also note that the fluidic channel anywhere outside the sensing region is assumed to have a height of $10 \mu \mathrm{m}$.

In the first step of our analysis, to decouple the interplay of diffusion, convection, and reaction, we have studied the transport of analyte molecules through the fluidic channels without considering binding in the sensing region (Figure $5 \mathrm{~d}, \mathrm{e})$. Under a low flow rate $\left(Q=1 \mu \mathrm{m}^{3} \cdot \mathrm{s}^{-1}\right)$, the analyte molecules are transported mainly by diffusion, which results in a slow increase of the mean concentration of the analyte molecules in the sensing region over time, $\bar{c}(t)=\int_{V} c / V$, where $V$ is the volume of the sensing region of the channel (Figure $5 \mathrm{~d}$ ). Increasing the flow rate results in faster transport, but shortens the time the analyte is present in the sensing region (Figure 5e). Furthermore, it can be seen that the analyte transport differs considerably for the microchannel and the nanochannel. For the microchannel, since the sensing volume is $100 \times$ higher than the sample volume, the maximum $\bar{c}(t)$ inside the sensing volume is $0.01 \times c_{0}$, which corresponds to a situation where $100 \%$ of the analyte molecules are present inside the sensing volume. On the other hand, for the nanochannel, since the volume of the channel is substantially smaller and matches the sample volume, the maximum $\bar{c}(t)$ is much higher $\left(0.3 \times c_{0}\right)$, and corresponds to a situation where maximal $30 \%$ of all of the analyte molecules are present inside the sensing volume. The rest of the molecules are "lost" due to diffusion into the channel outside the sensing region.

In the second step of our analysis, we now include the binding and unbinding of analyte molecules inside the sensing region. The corresponding results for a $k_{\text {on }}-k_{\text {off }}$ space characteristic of proteins are presented as the mean number of binding events for each molecule in the sample volume in Figure $6 a-d$. It has to be noted that the mean number of binding events for a nano- and microchannel with any applied flow cannot exceed the limits set by the previous case of the enclosed nano- and microwell, respectively, as described in Section 3 (added as dashed lines in Figure 6). At the beginning of the detection, the sample volume is localized mainly outside the sensing region (i.e., $\bar{c}$ is low, see Figure 5e), therefore the mean number of binding events for each molecule is considerably lower compared to the case of the enclosed fluidic wells. Subsequently, as time passes, more and more molecules are transported toward the sensing area in the channel, (i.e., $\bar{c}$ increases, see Figure 5e). Therefore the mean 

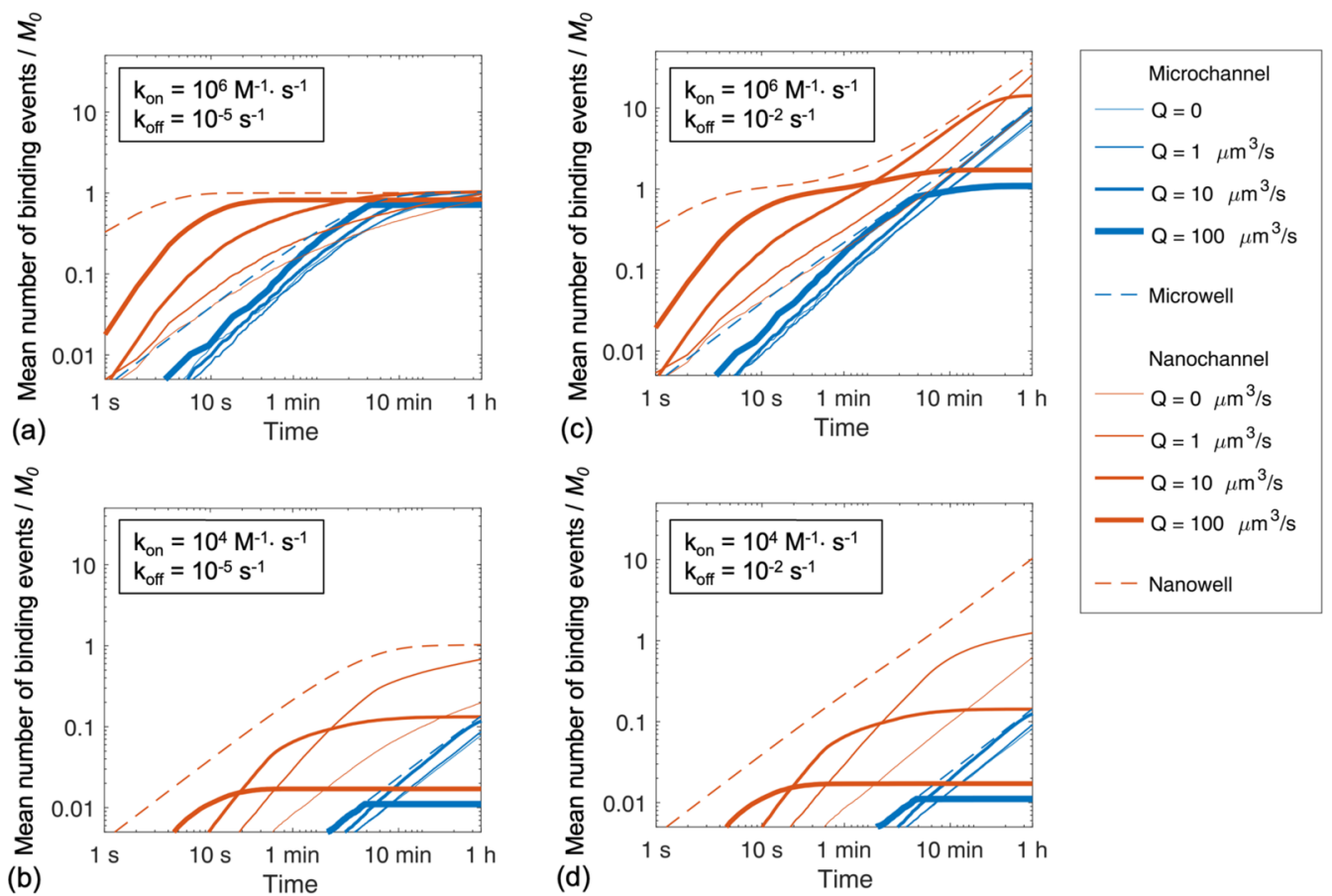

Figure 6. Mean number of binding events for each molecule present in a $1 \mathrm{pL}$ volume, $N^{+} / M_{0}$, detected inside the fluidic micro- or nanochannels depicted in Figure 5a,b, respectively, for a range of applied flow rates $Q=0-100 \mu \mathrm{m}^{3} \cdot \mathrm{s}^{-1}$. For comparison, the data from the enclosed fluidic microand nanowells depicted in Figure $3 \mathrm{c}$,e, respectively, are also shown. The results were obtained from stochastic simulations for a $k_{\text {on }}-k_{\text {off }}$ space characteristic of proteins.

number of binding events increases rapidly. However, it is overall lower compared to the case of the enclosed fluidic well due to the "loss" of molecules because of the applied flow. Increasing the flow rate results in an increase of the mean number of binding events at the beginning of the detection but at the cost of losing more molecules that never bind. Hence, the choice of the optimal flow rate is dependent on the binding kinetics of the specific molecules at hand and the required detection time. Specifically, for high $k_{\text {on }}$ and low $k_{\text {off }}$ (Figure $6 a)$, most binding events would be detected at a high flow rate, independent of the detection time. On the other hand, for the remaining cases (Figure $6 c, d$ ), for short detection times more binding events will be detected at high flow rates and for long detection times more binding events will be detected at low flow rates.

As the key result, we thus find that the overall binding characteristics in the nanofluidic channel (Figure 5b) can be improved by at least an order of magnitude compared to the microfluidic channel (Figure 5a) or the enclosed microwell (Figure 3e) for any specific type of binding kinetics or detection time. Therefore, we postulate that such a configuration presents an alternative to single-cell microfluidic devices that are used for the analysis of the extracellular environment and metabolite secretion ${ }^{21,22}$ or for lysis and the subsequent study of the intracellular content. ${ }^{34}$ To this end, two experimental studies suggesting single-cell analysis based on nanofluidic devices using fluorescent labeling ${ }^{8}$ or nanosampling ${ }^{7}$ exist.

\section{CONCLUSIONS}

In conclusion, our theoretical analysis has shown that decreasing the dimensions of a fluidic channel from the regime characteristic of microfluidics to the regime of nanofluidics can reduce the detection time from ultrasmall volumes (below hundreds of picoliters) by up to 2 orders of magnitude and thus make single-molecule detection practically viable. Specifically, the showcased representative example inspired by a single living cell with $1 \mathrm{pL}$ volume has shown that for the whole range of association and dissociation constants characteristic of affinity-based protein detection, most of the molecules in the sensing volume can be detected within minutes. Furthermore, for kinetics involving the creation of unstable complexes due to fast unbinding kinetics, we have demonstrated that every molecule can be detected multiple times within tens of minutes in a nanofluidic channel and thus improve the detection limit of the sensor considerably, thanks to this "molecular multiplier" effect. We therefore predict that nanoplasmonic sensors integrated with nanofluidics present a promising new paradigm for singlemolecule detection from ultrasmall sample volumes, such as single cells.

\section{METHODS}

6.1. Stochastic Diffusion-Convection-Reaction Simulations of Biomolecular Interactions. To describe the molecular binding rigorously, we have implemented stochastic diffusionconvection-reaction simulations. Our model is designed to mimic the behavior of a single molecule inside a fluidic structure with functionalized arrays of NPs. It is inspired by the previous work from the field of stochastic simulations of diffusion-controlled reactions ${ }^{35}$ and random walk simulations of convective-diffusive transport inside a fluidic structure. ${ }^{25}$

The Reynolds number is a dimensionless parameter that helps predict flow patterns in different fluid flow situations and it is defined as

$$
R e=\frac{Q D_{\mathrm{H}}}{A \nu}
$$


where $D_{\mathrm{H}}$ is the hydraulic diameter that for channels with a high aspect ratio is equal to twice the shorter axis of the channel, $A$ is the channel's cross-sectional area, and $\nu$ is the kinematic viscosity $(\nu=$ $10^{-3} \mathrm{~m}^{2} \cdot \mathrm{s}^{-1}$ for water). Since the Reynolds number pertinent to assumed geometries and the flow rates $\left(Q=0-100 \mu \mathrm{m}^{3} \cdot \mathrm{s}^{-1}\right)$ varies between 0 and $2 \cdot 10^{-7}$, which is far from the turbulent regime $(R e>$ 2000), only laminar flow can be considered here. Therefore, for the quasi-two-dimensional (2D) configuration $(W \gg H)$, we assume the axial fluid velocity to be

$$
v_{z}=\frac{6 Q}{W H^{3}} z(H-z)
$$

where $z$ is the vertical coordinate, which follows the $2 \mathrm{D}$ solution to the Navier-Stokes equations. For the configuration where $W \geq H$, a $2 \mathrm{D}$ velocity-field profile was calculated according to the analytical solution of the $3 \mathrm{D}$ Navier-Stokes equations for channels with a rectangular profile. $^{36}$

At time zero, $M_{0}$ molecules were randomly placed inside the defined volume and their movement inside the fluidic structure was recorded in time. At each time step, the position of each molecule was updated through the addition of a convective and diffusive component as

$$
\begin{aligned}
& x\left(t_{i+1}\right)=x\left(t_{i}\right)+R_{x}+v_{z} \Delta t \\
& y\left(t_{i+1}\right)=y\left(t_{i}\right)+R_{y} \\
& z\left(t_{i+1}\right)=z\left(t_{i}\right)+R_{z}
\end{aligned}
$$

where $R_{x}, R_{y}$, and $R_{z}$ were calculated separately at each time step as a normally distributed random number with a mean of zero and a standard deviation of $\sqrt{2 D \Delta t}$. The time step for all simulations was set to $\Delta t=10^{-5} \mathrm{~s}$, determined by convergence tests.

The surface of NPs was discretized in a way representing different molecular receptor locations, i.e., into patches with area $\Gamma_{0}^{-1}$. When the center of a molecule (a molecule of zero volume is assumed) hits the surface of a plasmonic NP at a specific point of time, it is decided based on the random number generation, whether the molecule binds or further diffuses into the volume of the fluidic structure. Specifically, if a random number $R<P_{\text {on }}$, where $P_{\text {on }}=1-\exp \left(k_{\text {on }} \Gamma_{0} \sqrt{\pi \Delta t / D}\right)$ is the probability of the binding, a molecule binds to the receptor and the time of the binding event is recorded. Further, in each time step it is decided if the molecule unbinds. Specifically, if a random number $R$ $<P_{\text {off }}$ where $P_{\text {off }}=1-\exp \left(k_{\text {off }} \Delta t\right)$ is the probability of unbinding, a molecule unbinds and the time of the unbinding event is also recorded. When the center of a molecule hits the wall of a fluidic structure, it is reflected back in a specular fashion. Thus, by repeating the process $M_{\text {sim }}$ times $\left(M_{\text {sim }}=10^{6}\right.$ for a single molecule present in 1 $\mathrm{pL}$ volume was used), we obtain characteristic histograms of subsequent binding and unbinding events, $n(t)$. The mean waiting time until the first binding event, $t_{1}$, is then calculated as the mean value of the first binding events detected for all molecules and the mean number of binding events collected over time is then calculated as

$$
N^{+}(t)=\frac{1}{M_{\text {sim }}} \int_{\tau=0}^{t} n(\tau)
$$

\section{ASSOCIATED CONTENT}

\section{SI Supporting Information}

The Supporting Information is available free of charge at https://pubs.acs.org/doi/10.1021/acssensors.0c01774.

Analytical model of the mass transport inside a fluidic channel containing an infinite volume of the target solution (Section 1) and stochastic simulations of the mass transport inside a fluidic well containing a finite volume of the target solution (Section 2) (PDF)
AUTHOR INFORMATION

\section{Corresponding Authors}

Barbora Špačková - Department of Physics, Chalmers University of Technology, 41296 Göteborg, Sweden;

Email: spackova@chalmers.se

Christoph Langhammer - Department of Physics, Chalmers University of Technology, 41296 Göteborg, Sweden; ○ orcid.org/0000-0003-2180-1379; Email: clangham@ chalmers.se

\section{Authors}

Hana Sípová-Jungová - Department of Physics, Chalmers University of Technology, 41296 Göteborg, Sweden; (1) orcid.org/0000-0002-5383-9120

Mikael Käll - Department of Physics, Chalmers University of Technology, 41296 Göteborg, Sweden; (1) orcid.org/00000002-1163-0345

Joachim Fritzsche - Department of Physics, Chalmers University of Technology, 41296 Göteborg, Sweden

Complete contact information is available at:

https://pubs.acs.org/10.1021/acssensors.0c01774

\section{Author Contributions}

The manuscript was written through contributions of all authors. All authors have given approval to the final version of the manuscript.

\section{Notes}

The authors declare no competing financial interest.

\section{ACKNOWLEDGMENTS}

This research has received funding from the Swedish Foundation for Strategic Research (FFL15-0087), the European Research Council (ERC) under the European Union's Horizon 2020 research and innovation program (678941/ SINCAT), and from the Knut and Alice Wallenberg Foundation project 2015.0055 .

\section{ABBREVIATIONS USED}

LSPR, localized surface plasmon resonance; NP, nanoparticle

\section{REFERENCES}

(1) Novotny, L.; Van Hulst, N. Antennas for Light. Nat. Photonics 2011, 5, 83

(2) Taylor, A. B.; Zijlstra, P. Single-Molecule Plasmon Sensing: Current Status and Future Prospects. ACS Sens. 2017, 2, 1103-1122.

(3) Beuwer, M. A.; Prins, M. W. J.; Zijlstra, P. Stochastic Protein Interactions Monitored by Hundreds of Single-Molecule Plasmonic Biosensors. Nano Lett. 2015, 15, 3507-3511.

(4) De Angelis, F.; Gentile, F.; Mecarini, F.; Das, G.; Moretti, M.; Candeloro, P.; Coluccio, M. L.; Cojoc, G.; Accardo, A.; Liberale, C.; Zaccaria, R. P.; Perozziello, G.; Tirinato, L.; Toma, A.; Cuda, G.; Cingolani, R.; Di Fabrizio, E. Breaking the diffusion limit with superhydrophobic delivery of molecules to plasmonic nanofocusing SERS structures. Nat. Photonics 2011, 5, 683-688.

(5) Seah, Y. F. S.; Hu, H. X.; Merten, C. A. Microfluidic single-cell technology in immunology and antibody screening. Mol. Aspects Med. 2018, 59, 47-61.

(6) Taniguchi, Y.; Choi, P. J.; Li, G. W.; Chen, H. Y.; Babu, M.; Hearn, J.; Emili, A.; Xie, X. S. Quantifying E-coli Proteome and Transcriptome with Single-Molecule Sensitivity in Single Cells. Science 2010, 329, 533-538.

(7) Lin, L.; Mawatari, K.; Morikawa, K.; Pihosh, Y.; Yoshizaki, A.; Kitamori, T. Micro/extended-nano sampling interface from a living single cell. Analyst 2017, 142, 1689-1696. 
(8) Xu, Y.; Jang, K.; Yamashita, T.; Tanaka, Y.; Mawatari, K.; Kitamori, T. Microchip-based cellular biochemical systems for practical applications and fundamental research: from microfluidics to nanofluidics. Anal. Bioanal. Chem. 2012, 402, 99-107.

(9) Li, X. K.; Soler, M.; Szydzik, C.; Khoshmanesh, K.; Schmidt, J.; Coukos, G.; Mitchell, A.; Altug, H. Label-Free Optofluidic Nanobiosensor Enables Real-Time Analysis of Single-Cell Cytokine Secretion. Small 2018, 14, No. 1800698.

(10) Špačková, B.; Lynn, N. S.; Slabý, J.; Sípová, H.; Homola, J. A Route to Superior Performance of a Nanoplasmonic Biosensor: Consideration of Both Photonic and Mass Transport Aspects. ACS Photonics 2018, 5, 1019-1025.

(11) Dahlin, A. B. Sensing applications based on plasmonic nanopores: The hole story. Analyst 2015, 140, 4748-4759.

(12) Sheehan, P. E.; Whitman, L. J. Detection limits for nanoscale biosensors. Nano Lett. 2005, 5, 803-807.

(13) Śípová, H.; Vrba, D.; Homola, J. Analytical Value of Detecting an Individual Molecular Binding Event: The Case of the Surface Plasmon Resonance Biosensor. Anal. Chem. 2012, 84, 30-33.

(14) Bocquet, L.; Charlaix, E. Nanofluidics, from bulk to interfaces. Chem. Soc. Rev. 2010, 39, 1073-1095.

(15) Fritzsche, J.; Albinsson, D.; Fritzsche, M.; Antosiewicz, T. J.; Westerlund, F.; Langhammer, C. Single Particle Nanoplasmonic Sensing in Individual Nanofluidic Channels. Nano Lett. 2016, 16, 7857-7864.

(16) Xu, Y.; Matsumoto, N.; Wu, Q.; Shimatani, Y.; Kawata, H. Sitespecific nanopatterning of functional metallic and molecular arbitrary features in nanofluidic channels. Lab Chip 2015, 15, 1989-1993.

(17) Shirai, K.; Mawatari, K.; Kitamori, T. Extended Nanofluidic Immunochemical Reaction with Femtoliter Sample Volumes. Small 2014, 10, 1514-1522.

(18) Weerakoon-Ratnayake, K. M.; Vaidyanathan, S.; Amarasekara, C. A.; Johnson, C. K.; Soper, S. A. Single Molecule Analysis in Nanofluidic Devices. In Spectroscopy and Dynamics of Single Molecules, 2019; Chapter 7.

(19) Feuz, L.; Jonsson, P.; Jonsson, M. P.; Hook, F. Improving the Limit of Detection of Nanoscale Sensors by Directed Binding to High-Sensitivity Areas. ACS Nano 2010, 4, 2167-2177.

(20) Mauriz, E.; Dey, P.; Lechuga, L. M. Advances in nanoplasmonic biosensors for clinical applications. Analyst 2019, 144, 7105-7129.

(21) Shirasaki, Y.; Yamagishi, M.; Suzuki, N.; Izawa, K.; Nakahara, A.; Mizuno, J.; Shoji, S.; Heike, T.; Harada, Y.; Nishikomori, R.; Ohara, O. Real-time single-cell imaging of protein secretion. Sci Rep. 2014, No. 4736.

(22) Oh, B. R.; Huang, N. T.; Chen, W. Q.; Seo, J. H.; Chen, P. Y.; Cornell, T. T.; Shanley, T. P.; Fu, J. P.; Kurabayashi, K. Integrated Nanoplasmonic Sensing for Cellular Functional Immunoanalysis Using Human Blood. ACS Nano 2014, 8, 2667-2676.

(23) Myszka, D. G.; Morton, T. A.; Doyle, M. L.; Chaiken, I. M. Kinetic analysis of a protein antigen-antibody interaction limited by mass transport on an optical biosensor. Biophys. Chem. 1997, 64, 127-137.

(24) Squires, T. M.; Messinger, R. J.; Manalis, S. R. Making it stick: convection, reaction and diffusion in surface-based biosensors. Nat. Biotechnol. 2008, 26, 417-426.

(25) Lynn, N. S.; Homola, J. Bio)Sensing Using Nanoparticle Arrays: On the Effect of Analyte Transport on Sensitivity. Anal. Chem. 2016, 88, 12145-12151.

(26) Wu, W. I.; Rezai, P.; Hsu, H. H.; Selvaganapathy, P. R. 1Materials and Methods for the Microfabrication of Microfluidic Biomedical Devices; Woodhead Publishing, 2013.

(27) Andersson, M.; Ek, J.; Hedman, L.; Johansson, F.; Sehlstedt, V.; Stocklassa, J.; Snogren, P.; Pettersson, V.; Larsson, J.; Vizuete, O.; Hjort, K.; Klintberg, L. Thin film metal sensors in fusion bonded glass chips for high-pressure microfluidics. J. Micromech. Microeng. 2017, 27, No. 015018

(28) Špačková, B.; Wrobel, P.; Bocková, M.; Homola, J. Optical Biosensors Based on Plasmonic Nanostructures: A Review. Proc. IEEE 2016, 104, 2380-2408.
(29) Dahlin, A. B. Size Matters: Problems and Advantages Associated with Highly Miniaturized Sensors. Sensors 2012, 12, 3018-3036.

(30) Collins, B. E.; Paulson, J. C. Cell surface biology mediated by low affinity multivalent protein-glycan interactions. Curr. Opin. Chem. Biol. 2004, 8, 617-625.

(31) Homola, J. Surface plasmon resonance sensors for detection of chemical and biological species. Chem. Rev. 2008, 108, 462-493.

(32) Hümmer, D.; Kurth, F.; Naredi-Rainer, N.; Dittrich, P. S. Single cells in confined volumes: microchambers and microdroplets. Lab Chip 2016, 16, 447-458.

(33) DeFronzo, R. A.; Ferrannini, E.; Keen, H.; Zimmet, P. Beta Cell Mass and Function in Human Type 2 Diabetes.; John Wiley \& Sons, Ltd.: New York, NY, 2015.

(34) Brown, R. B.; Audet, J. Current techniques for single-cell lysis. J. R. Soc., Interface 2008, 5, S131-S138.

(35) Claudio, V.; Dahlin, A. B.; Antosiewicz, T. J. Single-Particle Plasmon Sensing of Discrete Molecular Events: Binding Position versus Signal Variations for Different Sensor Geometries. J. Phys. Chem. C 2014, 118, 6980-6988.

(36) Wu, B.; Van Hirtum, A.; Luo, X. Y. Pressure Driven Steady Flow in Constricted Channels of Different Cross-Section Shapes. Int. J. Appl. Mech. 2013, 5, No. 1350002. 